\title{
Valoración de impacto ambiental por pesticidas agrícolas
}

\author{
Alejandro Alberto SCHAAF \\ Universidad Nacional deJujuy (Argentina) \\ CIT-Jujuy. Consejo Nacional de Investigaciones Científicas (CONICET) \\ schaaf.alejandro@gmail.com
}

Recibido: 10 de febrero del 2015

Enviado a evaluar: 15 de febrero del 2015

Aceptado: 25 de noviembre del 2015

\section{RESUMEN}

El uso de agroquímicos en Argentina se ha fortalecido junto con la expansión agrícola. El objetivo de este trabajo es la elaboración de una metodología para calcular el impacto ambiental que los pesticidas causan en el medio ambiente teniendo en cuenta los siguientes factores: "ecotoxicología": categoría toxicológica, toxicidad en abejas, aves y peces; "Toxicidad humana": carcinogenicidad, neurotoxicidad, alteraciones endocrinas, genotoxicidad y capacidad irritativa; "Comportamiento ambiental": la persistencia en el agua / sedimento, persistencia en el suelo y bioconcentración. Estos factores fueron clasificados como bajo, medio, alto o muy alto, de acuerdo con su nivel de toxicidad. Los resultados indican que los plaguicidas más tóxicos son los insecticidas. La mayoría de los pesticidas utilizados son la toxicidad media $(43,75 \%)$, seguido de baja y alta toxicidad $(21,88 \%)$ y, los de muy alta toxicidad $(12,5 \%)$. La metodología propuesta podría ser utilizada como una herramienta de monitoreo, gestión o educación ambiental.

Palabras clave: Agroquímicos, toxicidad, monitoreo ambiental.

\section{Environmental impact assessment of agricultural pesticides}

\begin{abstract}
The use of agrochemicals in Argentina has strengthened along with agricultural expansion. The aim of this paper is to draw up a methodology to calculate the environmental impact that pesticides cause in the environment considering the following factors: "ecotoxicology": toxicological category, toxicity to bees, birds and fish; "human toxicity": carcinogenicity, neurotoxicity, endocrine disruption, genotoxicity and irritative capacity; "environmental behavior": persistence in water / sediment, soil persistence and bioconcentration. These factors were then categorized as Low, Medium, High or Very High, according to their toxicity level. Results indicate that the most toxic pesticides are among the group of insecticides. Most of the pesticides used are middle toxicity (43.75\%), followed by high and low toxicity $(21,88 \%)$ and finally the very high toxicity $(12.5 \%)$. The proposed methodology could be used either as a monitoring tool, for management or environmental education.
\end{abstract}

Key words: Chemicals, toxicity, environmental monitoring. 


\section{Évaluation de l'impact environnemental des pesticides agricoles}

\section{RÉSUMÉ}

L'utilisation de produits agrochimiques en Argentine a été renforcée avec l'expansion agricole. L'objectif de ce travail est de développer une méthodologie pour le calcul de l'impact environnemental que les pesticides causent à l'environnement en tenant compte des facteurs suivants: "écotoxicologie" catégorie toxicologique, la toxicité pour les abeilles, les oiseaux et les poissons; "Toxicité humaine": cancérogénicité, neurotoxicité, perturbation du système endocrinien, la génotoxicité et la capacité irritative; "Performance environnementale": la persistance dans l'eau / sédiments, la persistance et la bioconcentration sol. Ces facteurs ont été classés comme faible, moyen, élevé ou très élevé, en fonction de leur niveau de toxicité. Les résultats indiquent que les pesticides sont des insecticides les plus toxiques. La plupart des pesticides utilisés sont la toxicité moyenne $(43,75 \%)$, suivie par la toxicité élevée et faible $(21,88 \%)$ et la toxicité très élevé $(12,5 \%)$. La méthodologie proposée pourrait être utilisé comme un outil de surveillance, de gestion ou de l'éducation environnementale.

Mots clés: Produits agrochimiques, la toxicité, la surveillance environnementale.

\section{INTRODUCCIÓN}

La Organización de las Naciones Unidas para la Alimentación y la Agricultura, mundialmente conocida como FAO (por sus siglas en inglés: Food and Agriculture Organization) establece que un plaguicida "es la sustancia o mezcla de ellas, destinada a prevenir, destruir o controlar plagas, incluyendo los vectores de enfermedad humana o animal; las especies no deseadas de plantas o animales que ocasionan un daño duradero $\mathrm{u}$ otras que interfieren con la producción, procesamiento, almacenamiento, transporte y comercialización de alimentos; los artículos agrícolas de consumo, la madera y sus productos, el forraje para animales o los productos que pueden administrárseles para el control de insectos, arácnidos u otras plagas corporales" (FAO, 1990). Independientemente de sus beneficios, es evidente que los plaguicidas son sustancias químicas deliberadamente tóxicas, creadas para interferir algún sistema biológico en particular y que carecen de selectividad real. Afectan simultáneamente, y en mayor o menor grado, tanto a la "especie blanco" como a otras categorías de seres vivos, particularmente al ser humano (Wania, 1998). En general se puede englobar al comportamiento ambiental de todas estas sustancias dentro de lo que se conoce como contaminación difusa, caracterizadas por no originarse en un punto definido, sino más bien en múltiples puntos, poco identificables. Este tipo de contaminación puede provocar situaciones especialmente preocupantes en el tiempo, ya que el ambiente puede ir cargándose de contaminantes, resultando afectadas extensas zonas (IARC, 2008).

Los plaguicidas se clasifican en función de algunas de sus características principales, como son la toxicidad aguda, la vida media, la estructura química y su uso (OMS/OPS, 1990). La exposición a plaguicidas puede afectar la salud de diversas formas. Efectos de deterioro de tipo funcional, lesiones patológicas que afectan el funcionamiento del organismo y reducen su capacidad de respuesta a factores de 
riesgo o estrés. De acuerdo con el tiempo de exposición para que se llegue a manifestar el efecto tóxico o de la duración del mismo, este puede ser de tipo agudo o crónico. Una vez emitido al ambiente (aire, agua, suelo y biota) el plaguicida tiene una dinámica y un destino propios, determinados no solo por sus propiedades físicas y químicas sino también por las características del medio con el que interactúa (Sors, 1987; OMS/OPS, 1990). Otros factores que intervienen con el comportamiento ambiental y la disponibilidad biológica de esta sustancia son el tipo de formulación; el método y las condiciones agrícolas en el momento de su aplicación. Lo anterior influye directamente en los efectos tóxicos sobre los organismos (terrestres y acuáticos) que se ven expuestos a esta sustancia. (Briggs, 1993; Walker et al., 2001)

Con respecto a Argentina, en los últimos 15 años la frontera agrícola se expandió de 15 a 30 millones de hectáreas cultivadas, generando cambios en el uso de la tierra en varias regiones del país, ocupando unos 2.8 millones de $\mathrm{km}^{2}{ }^{2}$ de tierras extraordinariamente fértiles que se ajustan perfectamente para la producción agrícola y ganadera (Viglizzo et al., 2006). A raíz de esto, el consumo de plaguicidas en nuestro país ha logrado consolidarse y esta consolidación ha fijado a dichos productos como uno de los pilares fundamentales del crecimiento de la producción en vistas de la evolución esperada a mediano plazo, que poseen un sinnúmero de variables influyentes, entre las cuales se pueden incluir variables relacionadas al mercado (precio, origen de los productos, oferta, etc.), variables relacionadas a los cultivos (mayor o menor incidencia de una adversidad durante un ciclo, características del genotipo, etc.), variables relacionadas a los productos (amplitud de control, nivel de sustitución, efectividad en el control, posibilidad de realizar mezclas), entre otras. (CASAFE, 2003).

Asumiendo las toxicidades, su impacto en el medio ambiente y en los seres vivos es que nos planteamos como objetivo de este trabajo diseñar una matriz de valoración que nos permita calificar el impacto ambiental de los diferentes pesticidas. El mismo es un estudio piloto desarrollado en la Provincia de Santa Fe, Argentina para ser utilizado como herramienta de monitoreo, gestión o educación ambiental.

\section{MATERIALES Y MÉTODOS}

Área de estudio. El área de estudio del proyecto se focalizó en la localidad de San Vicente, provincia de Santa Fe, Argentina, dentro del departamento Castellano (31 $\left.{ }^{\circ} 41^{\prime} 55.29^{\prime \prime} \mathrm{S}, 61^{\circ} 34^{\prime} 5.18^{\prime \prime O}\right)$. El clima de Santa Fe presenta dos gradientes, uno térmico de norte a sur, y otro hídrico de este a oeste. Por el régimen térmico el clima puede definirse como templado sin estación fría en el sur y templado y cálido en el norte; y por el régimen hídrico varía de húmedo a subhúmedo de este a oeste. El relieve es llano con una altura promedio de $40 \mathrm{~m}$ de altitud (Lewis \& Collantes 1974). La Provincia de Santa Fe es una de las más significativas productoras agrícolas del país, donde el sector agrícola representa aproximadamente el 65\% del Ingreso Bruto Total generado por el sector rural provincial. Con respecto al uso del suelo, se observa, en las últimas décadas, una expansión progresiva de la agricultura respecto de la ganadería. Pero la expansión agrícola actual no es del mismo tipo que la de hace 
tres o cuatro década atrás, ya que casi no queda superficie disponible de los mejores suelos; de aquí en más, la expansión factible sería por intensificación de la superficie actualmente en uso y por expansión sobre tierras de menor aptitud y mayor riesgo productivo. Los principales cultivos tanto para santa fe como para el área de estudio son soja, maíz, trigo, girasol y sorgo (MinAgri, 2010)

Para la recolección de información necesaria para obtener los diferentes tipos de pesticidas que se utilizan en la zona se utilizo un formato de encuesta. A partir de esto, durante el año 2012 se realizó un relevamiento de los tipos de cultivos y los diferentes pesticidas que se aplican con pulverizadoras terrestres en la zona agrícola de dicha localidad. Las consultas fueron realizadas a trabajadores del área, productores e ingenieros agrónomos. Para cada caso se les solicitó la siguiente información: cultivo, tipo de pesticida que se aplica con pulverizadora (nombre comercial y principio activo) y su acción o clase (fungicida, insecticida, etc.).

Con base en la matriz diseñada por Fernández et al. (2003) se diseño una escala que permitiera valorar las distintas características de las sustancias en relación a diferentes factores, siendo estos factores los referidos a salud y daños en el medio ambiente. Denominamos a esto, valoración de los impactos ambientales (V.I.A) y lo que buscamos con esto es tener un dato más amplio además de los datos de los propios productos comercializados. Permitiendo de esta manera tener una en el medio ambiente.

Factores considerados y sus correspondientes subfactores:

a) Ecotoxicología: categoría toxicológica, toxicidad en abejas, aves y peces.

b) Toxicidad humana: carcinogenicidad, neurotoxicidad, disrupción endocrina, genotoxicidad y capacidad irritativa.

c) Comportamiento ambiental: persistencia en agua/sedimento, persistencia en suelo y bioconcentración.

Después, estos factores fueron incluidos en los siguientes niveles de toxicidad:

$$
\text { Bajo - Medio - Alto - Muy alto. }
$$

A continuación se define cada subfactor considerado en la evaluación ambiental de los pesticidas según OMS/OPS (1990), IARC (2008) y EPA (2012):

1. Categoría toxicológica: distintas clases toxicológicas en las que pueden estar incluidos los pesticidas. Es decir, toxicidad aguda del ingrediente activo.

2. Toxicidad aves, abejas y peces: Capacidad de una sustancia química de causar daños en la estructura o funciones de los organismos vivos, o incluso la muerte.

3. Carcinogenicidad: Es la inducción de un crecimiento normal, desordenado y potencialmente ilimitado de las células de un tejido u órgano. 
4. Neurotoxicidad: Se refiere a efectos sobre el sistema nervioso central, el sistema nervioso periférico y los órganos de los sentidos.

5. Disrupción endocrina: Un disruptor endocrino es una sustancia química capaz de alterar el equilibrio hormonal y de provocar diferentes efectos adversos sobre la salud.

6. Genotoxicidad: Alteración en el material genético o en sus componentes asociados, producida por un agente químico en los niveles subtóxicos de exposición.

7. Capacidad irritativa: es la capacidad de una sustancia para producir una lesión, irritación o alergia a nivel de la piel, los ojos y las mucosas.

8. Persistencia en suelo y agua/sedimento: El concepto de persistencia a menudo se relacionado con el tiempo de permanencia de una sustancia química en el ambiente. A mayor tiempo de permanencia, mayor es la persistencia. La vida media $\left(\mathrm{DT}_{50}\right)$ de la sustancia es una medida de su persistencia.

9. Bioacumulación o bioconcentración: Estos son términos utilizados para describir el aumento en la concentración de una sustancia química, en un organismo en relación con la concentración de esa misma sustancia en el medio circundante.

En base al trabajo de Fernández et al. (2003) diseñamos la fórmula para determinar la valoración de los impactos. En el cuadro 1 se detalla cada uno de los valores que las mismas pueden tomar, la escala empleada:

\section{Fórmula $\quad \mathrm{VIA}=\left(\mathbf{E T} \mathrm{total}+\mathrm{TH}_{\text {total }}+\mathrm{CA}_{\text {total }}\right) \times 10$}

Donde:

$$
\begin{aligned}
& \mathbf{E T} \mathbf{t o t a l}_{\text {to }}=\mathrm{ET}_{1}+\mathrm{ET}_{2}+\mathrm{ET}_{3}+\mathrm{ET}_{4} \\
& \mathbf{T H} \text { total }=\mathrm{TH}_{1}+\mathrm{TH}_{2}+\mathrm{TH}_{3}+\mathrm{TH}_{4} \\
& \underbrace{\mathbf{C A}_{\text {total }}}_{\text {Factores }}=\underbrace{\mathrm{CA}_{1}+\mathrm{CA}_{2}+\mathrm{CA}_{3}+\mathrm{CA}_{4}}_{\text {Subfactores }}
\end{aligned}
$$


Cuadro 1. Escala de valores utilizada para cada una de las variables.

ECOTOXICOLOGÍA - ET

\begin{tabular}{ll}
\multicolumn{2}{c}{ CATEGORIAS TOXICOLÓGICAS $\left(\mathbf{E T}_{1}\right)$} \\
\hline IV Probablemente sin riesgo toxicológico & $\mathbf{1}$ \\
III Ligeramente tóxico & $\mathbf{2}$ \\
II Moderadamente tóxico & $\mathbf{4}$ \\
Ib Altamente tóxico & $\mathbf{6}$ \\
Ia Extremadamente tóxico & $\mathbf{8}$ \\
\multicolumn{1}{c}{ TOXICIDAD EN AVES $\left(\mathbf{E T}_{3}\right)$} & \\
\hline Practicamente no tóxico & $\mathbf{1}$ \\
Ligeramente tóxico & $\mathbf{2}$ \\
Moderadamente tóxico & $\mathbf{4}$ \\
Muy tóxico & $\mathbf{6}$ \\
Extremadamente tóxico & $\mathbf{8}$
\end{tabular}

\begin{tabular}{ll}
\multicolumn{2}{c}{ TOXICIDAD EN ABEJAS $\left.\left(\mathbf{E T}_{2}\right)\right)$} \\
\hline Virtualmente no tóxico & $\mathbf{1}$ \\
Ligeramente tóxico & $\mathbf{2}$ \\
Moderadamente tóxico & $\mathbf{4}$ \\
Altamente tóxico & $\mathbf{6}$
\end{tabular}

TOXICIDAD HUMANA - TH

\begin{tabular}{|c|c|c|c|}
\hline \multicolumn{2}{|c|}{ CARCINOGENICIDAD $\left(\mathbf{T H}_{1}\right)$} & \multicolumn{2}{|c|}{ DISRUPCIÓN ENDOCRINA ( TH $_{2}$ ) } \\
\hline No clasificable como cancerígeno & 1 & No existe & 1 \\
\hline Probablemente no cancerígeno & 2 & Sospecha & 3 \\
\hline Probablemente cancerígeno & 4 & Evidencia & 5 \\
\hline Carcinogénico para los seres humanos & 6 & & \\
\hline \multicolumn{2}{|c|}{ GENOTOXICIDAD $\left(\mathbf{T H}_{3}\right)$} & \multicolumn{2}{|c|}{ NEUROTOXICIDAD $\left(\mathbf{T H}_{4}\right)$} \\
\hline Negativo & 1 & Negativo & 1 \\
\hline Positivo & 5 & Positivo & 5 \\
\hline \multicolumn{2}{|c|}{ CAPACIDAD IRRITATIVA $\left(\mathbf{T H}_{5}\right)$} & & \\
\hline Negativo & 1 & & \\
\hline Positivo & 5 & & \\
\hline
\end{tabular}

COMPORTAMIENTO AMBIENTAL - CA

\begin{tabular}{lcccc}
\multicolumn{2}{c}{ PERSISTENCIA EN AGUA/SEDIMENTO $\left(\mathbf{C A}_{\mathbf{1}}\right)$} & & PERSISTENCIA EN SUELO $\left(\mathbf{C A}_{2}\right)$ \\
\cline { 1 - 2 } Baja $\left(\mathrm{DT}_{50}\right.$ días $\left.<60\right)$ & $\mathbf{1}$ & & No persistente $(<15$ días $)$ & $\mathbf{1}$ \\
Alta $\left(\mathrm{DT}_{50}\right.$ días $\left.>60\right)$ & $\mathbf{5}$ & & Ligera $(15-30$ días $)$ & $\mathbf{2}$ \\
\multicolumn{1}{c}{ BIOCONCENTRACIÓN $\left(\mathbf{C A}_{3}\right)$} & & & Media $(30-60$ días $)$ & $\mathbf{4}$ \\
\hline Ligera $(\mathrm{FBC}<100)$ & $\mathbf{1}$ & & Extra $(60-120$ días $)$ & $\mathbf{6}$ \\
Mediana $(\mathrm{FBC}: 100-1000)$ & $\mathbf{2}$ & & & $\mathbf{8}$ \\
Alta $(\mathrm{FBC}: 1000-5000)$ & $\mathbf{4}$ & & \\
Extrema $(\mathrm{FBC}>5000)$ & $\mathbf{6}$ & &
\end{tabular}

\begin{tabular}{|c|c|c|}
\hline \multirow{2}{*}{$\begin{array}{c}\text { VALORACIÓN IMPACTO } \\
\text { AMBIENTAL }\end{array}$} & BAJO & $\leq 200$ \\
\cline { 2 - 3 } & MEDIO & $\mathbf{2 0 0 - 3 5 0}$ \\
\cline { 2 - 3 } & ALTO & $350-450$ \\
\cline { 2 - 3 } & MUY ALTO & $\geq 450$ \\
\hline
\end{tabular}

Fuente: Elaboración propia. 
Obtención de los diferentes datos:

Ecotoxicología: estos datos se obtienen de las etiquetas de los pesticidas. Es información que viene plasmada en los productos por lo que es de fácil acceso. Se lo puede encontrar con el nombre de ficha técnica.

Toxicidad humana: estos datos se pueden extraer de diferentes fuentes: de la Agencia de Protección al Ambiente de los Estados Unidos (EPA) y la Agencia Internacional para la Investigación del Cáncer (IARC), a través de sus sitios web oficiales. Aunque también se pueden extraer de datos de laboratorios y universidades a través de informes toxicológicos de diferentes productos. En el apartado "capacidad irritativa" se toma en cuenta mucosa, piel y ojos; por lo tanto cuando una de estas se ve afectada se coloca 5 .

Comportamiento ambiental: estos datos se extraen de trabajos realizados por laboratorios, universidades, entidades, como así también de las etiquetas de los productos, en algunos casos se puede contar con esta información (sobre todo la de persistencia en el suelo).

Supuestos a tener en cuenta: cuando no se encontró información al respecto de alguna de las distintas categorías se procedió a colocar el menor valor, o sea 1 . Y se lo aclaro con un asterisco $\left(^{*}\right)$ en la matriz. En el caso de productos que vengan con más de un ingrediente activo, es decir, más de una sustancia, se procedió a analizar las dos por separado y colocar la de mayor impacto.

\section{RESULTADOS Y DISCUSIÓN}

Encontramos un total de 32 productos (pesticidas) utilizados en el área de estudio, de los cuales 15 son insecticidas, 10 son herbicidas y 7 fungicidas que se aplican con pulverizadoras. Los resultados de la valoración de impacto ambiental (V.I.A) de los diferentes pesticidas se detallan en el cuadro 2.

Con respecto a los resultados de este estudio preliminar observamos que la agricultura intensiva que actualmente se lleva a cabo en la localidad de San Vicente, provincia de Santa Fe requiere de una importante cantidad de pesticidas para asegurar el crecimiento y buen estado de los cultivos que allí se siembran, siendo la mayoría de estos insecticidas, seguido de los herbicidas y fungicidas (ver detalles Schaaf, 2013).

Tomando los valores del modelo diseñado se observa que la mayoría de los pesticidas utilizados son de mediana toxicidad $(43,75 \%)$, seguido por los de baja y alta toxicidad $(21,88 \%)$ y por último los de muy alta toxicidad $(12,5 \%)$. Con respecto a casos puntuales de los productos que se comercializan, los que deben permanecer en la mira, tanto por su categoría toxicológica como por sus altos valores de toxicidad (V.I.A), son los clorpirifos, endosulfan, fipronil, clap, cipermetrina, metamidofos, lufenuron, abamectina, 2-4 D, poseidon y lambda. Estos requieren de un seguimiento estricto debido a su alto y muy alto impacto en el medio ambiente, sobre todo si se trata de áreas donde existan poblaciones cercanas, ríos, criaderos de animales o apicultores. 
Cuadro 2. Cálculo y valoración final de los diferentes pesticidas utilizados en San Vicente, Santa Fe, Argentina.

\begin{tabular}{|c|c|c|c|c|c|c|c|c|c|c|c|c|c|c|c|}
\hline \multirow{2}{*}{ CLASE } & \multirow{2}{*}{$\begin{array}{l}\text { INGREDIENTE } \\
\text { ACTIVO }\end{array}$} & \multirow{2}{*}{$\begin{array}{l}\text { NOMBRES } \\
\text { COMERCIALES }\end{array}$} & \multicolumn{4}{|c|}{ ECOTOXICOLOGÍA } & \multicolumn{5}{|c|}{ TOXICIDAD HUMANA } & \multicolumn{3}{|c|}{$\begin{array}{l}\text { COMPORTAMIENTO } \\
\text { AMBIENTAL }\end{array}$} & \multirow{2}{*}{ V.I.A } \\
\hline & & & ET1 & ET2 & ET3 & ET4 & TH1 & TH2 & тH3 & TH4 & TH5 & CA1 & $\mathrm{CA} 2$ & $\mathrm{CA} 3$ & \\
\hline \multirow{15}{*}{$\begin{array}{l}\text { I } \\
\text { N } \\
\text { S } \\
\text { E } \\
\text { C } \\
\text { T } \\
\text { I } \\
\text { C } \\
\text { I } \\
\text { D } \\
\text { A } \\
\text { S }\end{array}$} & Abamectina & $\begin{array}{c}\text { ABAMECTINA AGM } \\
\text { HELM }\end{array}$ & 4 & 6 & 1 & 6 & 1 & $1^{*}$ & 3 & 5 & 5 & 5 & 4 & 1 & 410 \\
\hline & Carbaril & $\begin{array}{l}\text { CARBARIL } \\
\text { SEVIN } 85 \mathrm{~S}\end{array}$ & 4 & 1 & 2 & 2 & 4 & 5 & 3 & $1^{*}$ & 5 & 1 & 2 & 1 & 300 \\
\hline & Cipermetrina & $\begin{array}{c}\text { CIPERMETRINA agm } \\
\text { SHERPA }\end{array}$ & 4 & 6 & 1 & 2 & 4 & 5 & 3 & 5 & 5 & 1 & 6 & 4 & 460 \\
\hline & Clorantraniliprole & CORAGEN & 1 & 1 & 1 & 1 & 1 & 1 & 1 & 1 & 5 & 1 & 4 & 2 & 200 \\
\hline & Clorpirifos & CLORPIRIFOS & 4 & 6 & 6 & 4 & 1 & 5 & 3 & 5 & 5 & 1 & 6 & 4 & 500 \\
\hline & Endosulfan & $\begin{array}{c}\text { ENDOSULFAN } 35 \\
\text { MASTER }\end{array}$ & 6 & 4 & 4 & 8 & 4 & 5 & 5 & 5 & 1 & $1^{*}$ & 6 & 4 & 520 \\
\hline & Fipronil & CLAP 20 SC & 4 & 6 & 6 & 6 & 4 & $1^{*}$ & 3 & $1^{*}$ & 5 & 1 & 6 & 2 & 430 \\
\hline & Lambdacialotrina & $\begin{array}{c}\text { LAMBDA } \\
\text { GOLPE } 5 \text { EC }\end{array}$ & 6 & 6 & 1 & 8 & 1 & 5 & 3 & $1^{*}$ & 5 & 1 & 6 & 4 & 460 \\
\hline & Lufenuron & $\begin{array}{c}\text { SORBA@ 050 EC } \\
\text { MATCH }\end{array}$ & 1 & 2 & 1 & 6 & $1^{*}$ & $1^{*}$ & 5 & $1^{*}$ & 5 & 5 & 8 & 4 & 370 \\
\hline & Metamidofos & METAMIDOFOS AGM & 8 & 4 & 6 & 1 & 2 & 5 & $1^{*}$ & 5 & 5 & 1 & 1 & 1 & 390 \\
\hline & Metoxifenocide & INTREPID & 1 & 1 & 1 & 1 & 1 & $1^{*}$ & $1^{*}$ & $1^{*}$ & 5 & $1^{*}$ & 6 & 1 & 170 \\
\hline & Metsulfuron & $\begin{array}{c}\text { METSULFURON } 60 \\
\text { Zamba }\end{array}$ & 1 & 1 & 1 & 1 & 1 & $1^{*}$ & $1^{*}$ & $1^{*}$ & 5 & 5 & 2 & 1 & 180 \\
\hline & $\begin{array}{c}\begin{array}{c}\text { Profenofos + } \\
\text { Lufenuron }\end{array} \\
\end{array}$ & CURYOM & 4 & 6 & 2 & 4 & 1 & 5 & $1^{*}$ & $1^{*}$ & 5 & $1^{*}$ & 1 & 1 & 290 \\
\hline & Tiametoxam & ACTARA & 1 & 6 & 1 & 1 & 1 & $1^{*}$ & $1^{*}$ & $1^{*}$ & 1 & 1 & 6 & 1 & 190 \\
\hline & Thiacloprid & CALIPSO $48 \mathrm{SC}$ & 4 & 4 & 6 & 4 & 4 & 5 & 1 & 1 & 1 & 1 & 2 & 1 & 340 \\
\hline \multirow{10}{*}{$\begin{array}{l}\text { H } \\
\text { E } \\
\text { R } \\
\text { B } \\
\text { I } \\
\text { C } \\
\text { I } \\
\text { D } \\
\text { A } \\
\text { S }\end{array}$} & $\begin{array}{l}\text { Acetamida: } 2 \text { - } \\
\text { cloroacetanilida }\end{array}$ & ACETOCLOR & 1 & 1 & 2 & 4 & 4 & $1^{*}$ & 5 & $1^{*}$ & 5 & $1^{*}$ & 1 & 1 & 240 \\
\hline & Atrazina & ATRASINA & 1 & 1 & 1 & 2 & 1 & 5 & 5 & $1^{*}$ & 5 & 5 & 4 & 1 & 310 \\
\hline & Clorimuron & 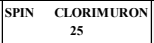 & 1 & 1 & 1 & 1 & $1^{*}$ & $1^{*}$ & $1^{*}$ & $1^{*}$ & 1 & $1^{*}$ & 4 & 1 & 100 \\
\hline & $2-4 \mathrm{D}$ & 2- - 4 D AGM & 4 & 4 & 4 & 2 & 1 & 5 & 5 & 5 & 5 & 1 & 4 & 1 & 410 \\
\hline & Dicamba & DICAMBA & 4 & 1 & 2 & 1 & 1 & 1 & $1^{*}$ & $1^{*}$ & 5 & 1 & 1 & 1 & 180 \\
\hline & Glifosato & ROUNDUP & 1 & 1 & 1 & 2 & 1 & 1 & 3 & 5 & 5 & 1 & 4 & 1 & 260 \\
\hline & Glufosinato de amonio & LIBERTY & 4 & 2 & 2 & 2 & $1^{*}$ & 1 & 3 & $1^{*}$ & 5 & 1 & 1 & 1 & 220 \\
\hline & Imazetapir & $\begin{array}{c}\text { PIVOT } \\
\text { ALTEZA }\end{array}$ & 1 & 4 & 1 & 1 & 1 & $1^{*}$ & $1^{*}$ & $1^{*}$ & 5 & $1^{*}$ & 4 & 1 & 180 \\
\hline & Metolacloro & BICEP @ PACK GOLD & 2 & 2 & 4 & 4 & 4 & $1^{*}$ & $1^{*}$ & $1^{*}$ & 5 & 5 & 1 & 2 & 290 \\
\hline & Paraquat & GRAMOXONE & 4 & 1 & 1 & 2 & 1 & 1 & 3 & 5 & 5 & 1 & 8 & 1 & 330 \\
\hline \multirow{7}{*}{$\begin{array}{l}\text { F } \\
\text { U } \\
\text { N } \\
\text { G } \\
\text { I } \\
\text { C } \\
\text { I } \\
\text { D } \\
\text { A } \\
\text { S }\end{array}$} & $\begin{array}{l}\text { Azoxistrobina + } \\
\text { Cyproconazole }\end{array}$ & AMISTAR & 4 & 1 & 4 & 4 & 2 & 1 & $1^{*}$ & $1^{*}$ & 5 & 5 & 6 & 1 & 330 \\
\hline & Carbendazim & CARBENDAZIM & 1 & 1 & 4 & 4 & 4 & 1 & 5 & 5 & 5 & 1 & 2 & 1 & 340 \\
\hline & Ditiocarbamato & CHEMISPOR & 1 & 4 & 1 & 1 & 1 & 5 & 3 & 5 & 5 & 1 & 1 & 2. & 300 \\
\hline & Mancozeb & MANCOZEB 75 DVA & 1 & 1 & 1 & 4 & 4 & 5 & 3 & 5 & 5 & 5 & 1 & 1 & 360 \\
\hline & Picoxystrobin & ACAPELA & 2 & 1 & 1 & 6 & 1 & 1 & 1 & $1^{*}$ & 1 & 5 & 6 & $1^{*}$ & 250 \\
\hline & Tebuconazol & TEBUCONAZOLE 43 & 1 & 1 & 1 & 4 & 4 & $1^{*}$ & 3 & $1^{*}$ & 5 & 5 & 6 & 2 & 320 \\
\hline & $\begin{array}{l}\text { Trifloxystrobin } \\
\text { Propiconazole }\end{array}$ & POSEIDON & 2 & 2 & 2 & 6 & 4 & 1 & 3 & $1^{*}$ & 5 & 5 & 4 & 2 & 360 \\
\hline
\end{tabular}

\begin{tabular}{|c|c|c|}
\hline \multirow{3}{*}{$\begin{array}{c}\text { VALORACIÓN IMPACTO } \\
\text { AMBIENTAL }\end{array}$} & BAJO & $\leq 200$ \\
\cline { 2 - 3 } & MEDIO & $200-350$ \\
\cline { 2 - 3 } & ALTO & $350-450$ \\
\cline { 2 - 3 } & MUY ALTO & $\geq 450$ \\
\hline
\end{tabular}

Fuente: Elaboración propia. 
La herramienta de valoración diseñada para este estudio, puede resultar de interés para el diagnostico y gestión ambiental del uso de pesticidas que se aplican con pulverizadoras. Cuenta con un amplio uso de factores donde se toma en cuenta la toxicidad en humanos, otros seres vivos, el impacto en el agua y en el suelo. La problemática de los agroquímicos en zonas de cultivo intensivo, no se debe remitir solamente a los posibles problemas de toxicidad sobre el ser humano, a pesar de ser este el tema de mayor preocupación (Fernández et al., 2003).

Los criterios adoptados y usados en esta metodología fueron desarrollados por el autor en base a trabajos posteriores, por lo que puede estar sujeta a cambios y mejoras en esta resultarían de ventajas para tener un panorama amplio de la contaminación ambiental en zonas agrícolas. Nos referimos a amplios aspectos y de forma general, por lo que, la misma debe ser implementada como base de monitoreo y estudios, recomendando investigaciones posteriores más detalladas y estrictos a la hora de tomar decisiones con respecto a que pesticidas recomendar, utilizar, sacar del mercado o prohibir. También se podría desarrollar para trabajos de educación ambiental en diferentes ámbitos, ya que cuenta con aspectos generales de contaminación por pesticidas en zonas agrícolas. La búsqueda bibliográfica y la familiarización con cada uno de los productos resultarían en una mirada amplia sobre los daños que estos causan al medio ambiente. La industria de los agroquímicos se encuentra en permanente avance, en donde todos los años surgen nuevos productos en el mercado, por lo que se hace necesario el relevamiento y monitoreo de pesticidas año tras año para un manejo adecuado de una región determinada, requiriendo la coordinación entre los gobiernos locales, pobladores, investigadores y el compromiso de los agricultores (Schaaf, 2013). Coincidiendo con lo afirmado por Fernández et al. (2003) se espera que en el futuro se puedan mejorar y profundizar tanto la metodología empleada como los resultados obtenidos, debido a la totalidad de factores considerados.

\section{BIBLIOGRAFÍA}

BRIGGS, S.A. (1993). Basic Guide to Pesticides: Their Characteristics and Hazards. Hemisphere Publishing Corporation, EE. UU.

CASAFE - CÁMARA DE SANIDAD AGROPECUARIA Y FERTILIZANTES. (2003). Guía de productos fitosanitarios de la República Argentina. Volumen 2.

EPA. AGENCIA DE PROTECCIÓN AMBIENTAL DE LOS ESTADOS UNIDOS. (2012). Integrated Risk Information System. Disponible en: http://www.epa.gov

FAO. (1990). Código Internacional de Conducta para la Distribución y Utilización de Plaguicidas. Roma. $40 \mathrm{p}$.

FERNÁNDEZ, N., VICIANA, V., \& DROVANDI, A. (2003). Valoración del impacto ambiental total por agroquímicos en la cuenca del río Mendoza. Proyecto OEI/DGI Barcala y Av. España (5500). Mendoza, Argentina.

IARC. INTERNATIONAL AGENCY FOR RESEARCH ON CANCER. (2008). Agent Reviewed by the IARC Monographs. Volumes 1-99, May. 
LEWIS JP y MB COLLANTES. (1974). La vegetación de la provincia de Santa Fe. Reseña general y enfoque del problema. Boletín de la Sociedad Argentina de Botánica 15: 343-356.

MINISTERIO DE AGRICULTURA, GANADERÍA Y PESCA DE LA REPÚBLICA ARGENTINA (MINAGRI). (2010). Disponible en: www.minagri.gob.ar.

ORGANIZACIÓN MUNDIAL DE LA SALUD (OMS), ORGANIZACIÓN PANAMERICANA DE LA SALUD (OPS), CENTRO PANAMERICANO DE ECOLOGÍA HUMANA Y SALUD. (1990). Serie Vigilancia, 9. Plaguicidas organoclorados. México: OMS/OPS.

SCHAAF, A. A. (2013). Uso de pesticidas y toxicidad: relevamiento en la zona agrícola de San Vicente, Santa Fe, Argentina. Revista mexicana de ciencias agrícolas, 4(2), 323-331.

SORS. (1987). Monitoreo ambiental. Instituto Nacional de Ecología. México

VIGLIZZO, ERNESTO F. \& JOBBÁGY ESTEBAN. (2006). Expansión de la Frontera Agropecuaria en Argentina y su Impacto Ecológico-Ambiental. Instituto Nacional de Tecnología Agropecuaria - INTA.

WALKER, C.E., HOPKIN, S.P., SIBLY, R.M., PEAKALL, D.B. (2001). Principles of Ecotoxicology. Second Edition. Taylor and Francis.

WANIA F. (1998). Multi-compartmental models of contaminant fate in the en 